\title{
Korrektureingriffe bei fehlverheilter distaler Radiusfraktur
}

\author{
T. Uzdil, K. H. Winker
}

\section{Zusammenfassung}

Ausgeheilte Fehlstellungen am handgelenknahen Unterarm stören ein komplexes biomechanisches Gefüge zwischen dem distalen Radius, dem Ulnakopf und der Handwurzel. Die Korrektur dieser Fehlstellung sollte bei entsprechendem Ausmaß mit zu erwartenden arthrotischen Spätfolgen und hinreichendem Leidensdruck des Patienten Bestandteil des Behandlungskonzeptes von distalen Radiusfrakturen sein. Die zur Verfügung stehenden Verfahren sehen ein additives Vorgehen durch Einfügen von Knochenkeilen am distalen Radius sowie ein subtraktives bzw. resezierendes
Vorgehen an der Ulna vor. Komplexe Fehlstellungen können auch durch Kombination von Verfahren behandelt werden. Neben der knöchernen Inkongruenz sollte eine deutliche Einschränkung der Unterarmdrehung, anhaltende Schmerzen, eine Kraftminderung und die Ästhetik die Indikation zur Korrektur mitbestimmen. Fortgeschrittene Arthrosen, Stufen in den Gelenkflächen, bestehende Weichteilschäden, Reflexdystrophien und die Altersosteoporose stellen dagegen Kontraindikationen dar. Als optimaler Zeitpunkt für Korrektureingriffe wird in der Literatur der 6.bis 9. posttraumatische Monat benannt.

\section{Einleitung}

Trotz zahlreicher Behandlungsoptionen und der Weiterentwicklung von Behandlungskonzepten ist die distale Radiusfraktur in vielen Fällen eine Problemfraktur. Die Fehlstellung nach Ausheilung zählt dabei zu den häufigsten Behandlungskomplikationen. In der Literatur werden Fehlstellungen nach handgelenknaher Radiusfraktur bei konservativer Behandlung in bis zu 74\% der Fälle beschrieben. Als Ursache für ausgeheilte Fehlstellungen am distalen Radius wird die unzureichende Primärreposition, ein sekundärer Korrekturverlust nach Reposition oder ein Fehlwachstum nach Epiphysenverletzung beim Kind genannt. Häufig werden auch durch übersehene oder unterbewertete Begleitverletzungen im Bereich der Handwurzel und des Ulnakopfes ungeeignete Therapieverfahren gewählt (Abb.1).

OP-JOURNAL 2000; 16: 35-39

(C) Georg Thieme Verlag Stuttgart · New York

\section{Biomechanische Überlegungen}

Beobachtet werden insbesondere Radiusverkürzungen mit relativem Ulnavorschub, die dreidimensionale Verkippung der Radiusbasis sowie Torsionsfehlstellungen oder Fehlstellungskombinationen.

Diese Verletzungs- bzw. Fehlstellungsmuster stören ein komplexes biomechanisches Gefüge aus distalem Radius, Ulnakopf und Handwurzel, wobei den Veränderungen im distalen Radioulnargelenk (DRUG) eine Schlüsselrolle zukommt [7].

So machen Inkongruenzen im DRUG Kontaktflächenveränderungen zwischen Incisura ulnaris radii und der korrespondierenden Circumferentia articularis ulnae [2]. Als Folge kommt es in stabilisierenden Strukturen des triangulären fibrokartilaginären Komplexes (TFCC), insbesondere am Ligamentum radioulnare dorsale sowie am Ligamentum radioulnare palmare, bei Drehbewegungen zum Spannungsanstieg bis hin zu einem mechanischen Impingement [1]. Resultierend entsteht eine erhöhte Druckbelastung

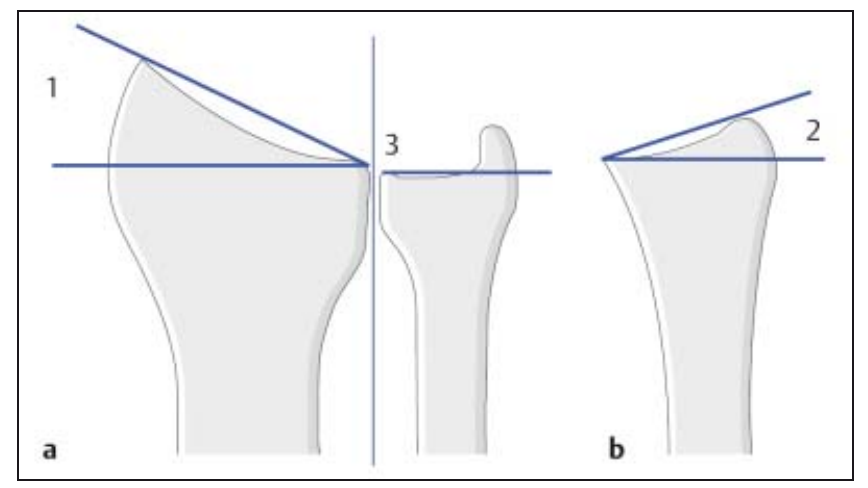

Abb.1 Physiologische Gelenkverhältnisse am distalen Unterarm. (a) Ansicht im a.p. Strahlengang mit Darstellung des radial offenen Speichenschaftgelenkwinkels (1) von ca. $30^{\circ}$ und der axialen radioulnaren Längenverhältnisse (3). (b) Ansicht im seitlichen Strahlengang mit Darstellung des dorsal offenen Speichenschaftgelenkwinkels (2) von ca. $10^{\circ}$. 
im DRUG und im ulnokarpalen Gelenkkompartment. Diese Abweichungen von der physiologischen Gelenk- und Bandarchitektur erzeugen klinisch überwiegend eine ulnarseitige Schmerzsymptomatik und Rotationseinschränkungen. Insbesondere die Supinationseinschränkungen werden im täglichen Leben als äußerst störend empfunden [5].

Verkippungen der Radiusbasis nach dorsal, palmar oder radial bewirken eine Fehlbelastung der distalen Radiusgelenkfläche, des Os lunatum sowie des Os scaphoideum und stellen bei hinreichender Ausprägung eine Präarthrose dar. Zunehmende posttraumatische Gelenkveränderungen und progrediente Schmerzen prägen dann den weiteren Verlauf.

So folgt zum Beispiel das Lunatum dem distalen Radius bei Extensionsfehlstellungen und unterliegt dadurch einer vermehrten Dorsalrotation, die vom Kapitatum und vom Skaphoid ausgeglichen werden muss. Die Folge sind Störungen der karpalen Kinematik bis hin zur mediokarpalen Instabilität [8]. Bewegungseinschränkungen der Dorsalextension und Palmarflexion werden subjektiv von vielen Patienten als weniger hinderlich bewertet.

\section{Indikation zur Korrektur}

Die Indikation zu Korrektureingriffen am distalen Unterarm sollte sich am Ausmaß der Fehlstellung, an zu erwartenden Spätfolgen sowie am Leidensdruck der Patienten orientieren.

Insbesondere Radiusverkürzungen über $6 \mathrm{~mm}$ und komplexe Fehlstellungen im Bereich des distalen Radius sollten einer Revision zugeführt werden. Aber auch isoliert auftretende Dorsal- und Palmarverkippungen der Radiusbasis werden bei entsprechender Ausprägung und resultierender Bewegungseinschränkung korrigiert. Das alleinige Auftreten einer sichtbaren Deformierung des Oberflächenreliefes sollte keine Indikation zu Korrekturosteosynthesen darstellen (Abb. 2-4).

Kontraindikationen zur Korrekturoperation sind Stufenbildungen in der distalen Radiusgelenkfläche, bereits fortgeschrittene Arthrosen und vorbestehende Weichteilschäden. Eine abgelaufene Reflexdystrophie, ein hohes biologisches Alter und Altersosteoporose werden ebenfalls als Kontraindikationen benannt.
Indikation bei entsprechendem

Fehlstellungsausmaß:

- deutliche Bewegungs-Einschränkung insbesondere der Supination/ Pronation

- Kraftminderung

Schmerzen

Ästhetik

Kontraindikation:

- fortgeschrittene Arthrose

- Stufen in der distalen Radiusgelenkfläche

- bestehender Weichteilschaden

- abgelaufene Reflexdystrophie

- Altersosteoporose

hohes Alter

\section{Vorbereitung und Durchführung}

Zur Korrektur von Fehlstellungen oder Fehlstellungskombinationen stehen verschiedene Verfahren zur Verfügung:

Verwendet werden isolierte Korrekturosteotomien am distalen Radius, kombinierte Korrekturen von Radius und Ulna, isolierte Ulnaverkürzungsosteotomien, die Reposition von Luxationen und Subluxationen sowie die Refixation des TFCC.

Generell unterscheidet man ein additives (Einfügen eines Knochenkeiles z.B. vom Beckenkamm) und subtraktives (Entnahme einer Knochenscheibe z.B. aus der Elle) Vorgehen.

Die Wahl des Verfahrens sollte nach eingehender Analyse des Röntgenbildes unter Berücksichtigung von ulnokarpalen Begleitverletzungen erfolgen. Eine OPPlanskizze ist bei der Vorbereitung des Eingriffes obligat und kann zur Patientenaufklärung verwendet werden.

Die Operation wird in der Regel in Allgemeinnarkose unter Verwendung eines Armtisches bei Rückenlage des Patienten und mit einer Oberarmblutsperre durchgeführt. Die Desinfektion und sterile Patientenabdeckung wird so plaziert, dass die Beckenregion zur Gewinnung eines kortikospongiösen Spanes zugänglich bleibt.

OP-Vorbereitung:

- Rückenlage

- Armtisch

- Blutsperre

- Bildverstärker

- Zugangsmöglichkeit zum Beckenkamm

\section{Verfahren}

Bei isolierten Korrekturoperationen am distalen Radius wird additiv nach Osteotomie ein kortikospongiöser Knochenspan eingebracht und mit einer T-Platte stabilisiert (CAMPBELL 1937).

Dies ist über den palmaren Zugang (bei Fehlstellung nach Flexions- und Extensionsfrakturen) in Supinationsstellung des Unterarmes möglich. Nach Hautschnitt von der distalen Handgelenkbeugefurche über der Sehne des M. flexor carpi radialis etwa $10 \mathrm{~cm}$ nach proximal erfolgt die Spaltung der Unterarmfaszie. Die Sehne des M. flexor carpi radialis wird nach ulnar verschoben, so dass der M. flexor digitorum superficialis und M. flexor pollicis longus zur Darstellung kommen. Diese werden nach ulnar verlagert, damit der darunter liegenden M. pronator quadratus vom Radius nach ulnar abgeschoben werden kann. Besondere Aufmerksamkeit verdienen der N. medianus und die A. radialis (Abb.5 u.6).

Der dorsale Zugang (heute seltener angewendet) wird nach Pronation des Unterarmes über eine gerade oder S-förmigen Hautinzision realisiert. Unterarmfaszie und Retinaculum extensorum werden nach präfaszialer Ablösung der Subkutis geradlinig über dem vierten Strecksehnenfach gespalten. Die Sehnen des M. extensor digitorum werden in Richtung Ulna verlagert, so dass ulnar der Sehne des M. extensor pollicis longus und des $\mathrm{N}$. interosseus posterior der Radius und die Gelenkkapsel zugänglich sind. In der Regel wird vor Korrekturosteotomie das Tuberculum dorsale radii abgemeißelt.

Radiale aber auch palmare Abkippungen der distalen Radiusgelenkfläche lassen sich über den Zugang von radial-palmar nach Inzision entlang der Beugefalte mit L-förmiger Fortführung nach proximal realisieren. Der Zugang zum Radius ist dann nach Eröffnung des ersten Strecksehnenfaches zwischen erster Strecksehne und M. flexor pollicis longus möglich. Die Korrektur erfolgt ebenfalls additiv mit einem entsprechenden Knochenspan nach Radiusosteotomie.

Der relative Ulnavorschub bei Radiusverkürzungen ohne wesentliche Verkippung oder Verdrehung der Radiusgelenkfläche kann über isolierte subtraktive Eingriffe an der Ulna korrigiert werden.

Dabei wird die Verkürzungs- bzw. Dekompressionsosteotomie nach Pechlaner 


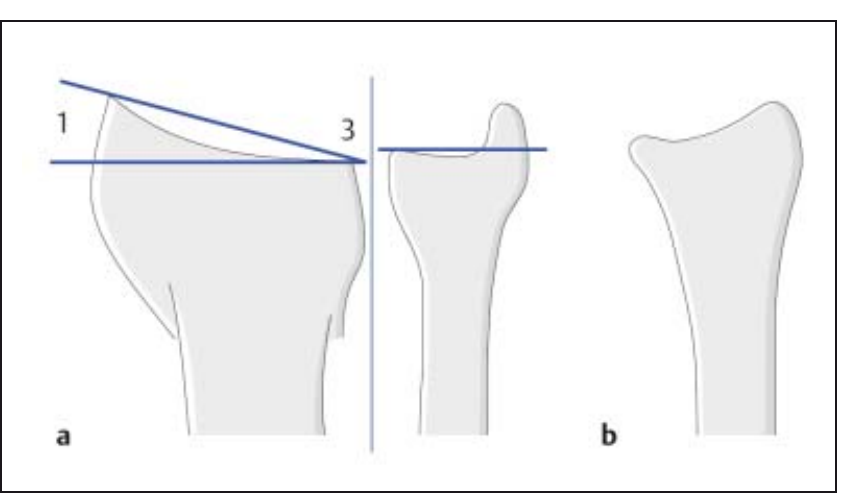

Abb. 2 Fehlstellung des distalen Speichenendes durch axiale Einstauchung mit relativem Ulnavorschub (3) und Abflachung des radial offenen Speichenschaftgelenkwinkels (1).

Abb. 3 Fehlstellung des distalen Speichenendes im seitlichen Strahlengang durch Palmarkippung der distalen Speichengelenkfläche.

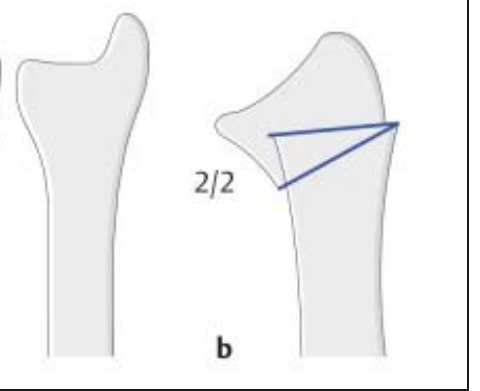

a

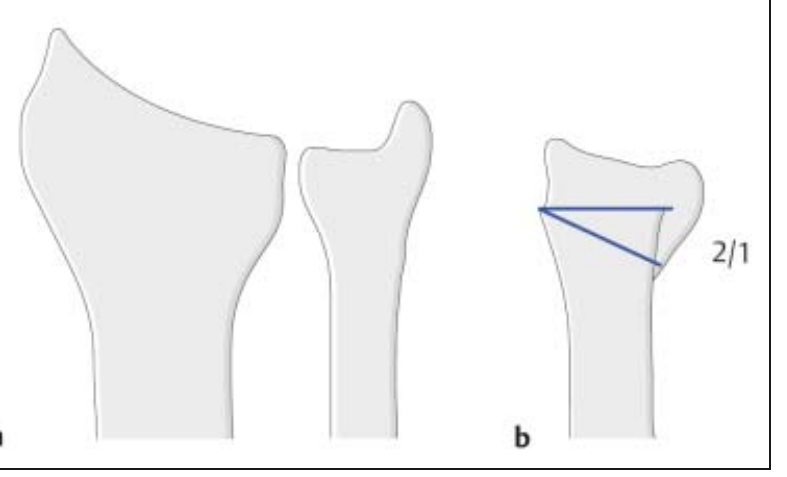

Abb. 4 Fehlstellung des distalen Speichenendes im seitlichen Strahlengang durch Dorsalkippung der distalen Speichengelenkfläche.

durch eine schräge Doppelsägenosteotomie am Ulnakopf von distal-radial nach ulnar-proximal unter Schonung der Gelenkfläche realisiert. Vorbedingung ist eine schmerzfreie Unterarmdrehbeweglichkeit und ein Ulnavorschub von mehr als $3 \mathrm{~mm}$.

Der Zugang verläuft S-förmig von dorsalulnar über dem distalen Ende der Elle. Nach Darstellung und Schonung des R. dorsalis nervi ulnaris wird die Sehne des $M$. extensor carpi ulnaris aus dem 6. Strecksehnenfach gelöst und die distale Ulna sowie das distale Radio-Ulnargelenk dargestellt.

Als weitere Verfahren stehen die Ulnasegmentresektion nach Kapandji und die ebenfalls schräge von distal-radial nach ulnar-proximal laufende Verkür- zungsosteotomie mit interfragmentärer Zugschrauben- oder mit Plattenosteosynthese zur Verfügung. Die Indikation für diese beiden Eingriffe sollte über eine isolierte axiale Radiusfehlstellung nicht hinaus gehen.

Die Resektion des Ulnakopfes nach Darrach wird nur bei Patienten mit schmerzhafter, deutlich eingeschränkter Unterarmdrehbeweglichkeit ohne körperliche Belastung und einem Alter über 50 Jahre durchgeführt. Der Zugang zur distalen Ulna wird durch Längsinzision zwischen den Sehnen des M. extensor carpi ulnaris und dem M. extensoris digiti minimi realisiert.

Bei komplexen Fehlstellungen, aber auch bei Fehlwachstum im Kindesalter, lassen sich subtraktive und additive Verfahren kombinieren, wobei primär die Radiuskorrektur und sekundär die Ulnakorrektur erfolgen sollte.

Dreidimensionale Fehlstellungen der Radiusbasis mit gleichzeitiger Luxation des Ulnakopfes oder einer länger bestehenden Fehlstellung im DRUG, die sich durch isolierte Radiusosteotomien nicht korrigieren lassen, sollten gleichzeitig oder besser sekundär durch eine Ulnakopfhemiresektion nach Bowers behandelt werden.

Bei ausgeprägter Begleitinstabilität im DRUG, die nach Radiuskorrekturosteotomie fortbesteht, empfiehlt es sich, das DRUG zu revidieren und gegebenenfalls den Processus styloideus ulnae mit einer Zuggurtung oder den TFCC mit einer transossären Naht zu refixieren [7].

Ist es bereits zu massiven arthrotischen Veränderungen im DRUG gekommen, sind rekonstruktive Eingriffe nicht mehr indiziert. Dann kommen Arthrodesen im DRUG mit gleichzeitiger distaler Ulnasegmentresektion nach Kapandji-Sauvi sowie Arthrodesen und Denervationen im Handgelenk in Frage. Diese Eingriffe sollten jedoch als Rettungsoperationen verstanden werden.

Additive Korrekturosteotomie

am Radius:

- mit kortikospongiösem Knochenspan von dorsal

- mit kortikospongiösem Knochenspan von palmar

- mit kortikospongiösem Knochenspan von radial

Subtraktive Korrekturosteotomie an der Ulna:

- Ulnasegmentresektion schräge Verkürzungsosteotomie am Ulnaschaft

- Dekompressionsosteotomie am Ulnakopf Ulnakopfresektion

Additive und subtraktive Kombinationen

\section{Nachbehandlung}

Postoperativ wird in der Regel ein zirkulärer Unterarmgips oder eine volare Unterarmgipsschiene für 3 Wochen angelegt.

Nach Gipsentfernung sollte durch entsprechende Analgesie eine schmerzfreie physio- bzw. ergotherapeutische Nachbehandlung erfolgen.

Eine Materialentfernung wird nach dorsaler Plattenosteosynthese regelhaft 


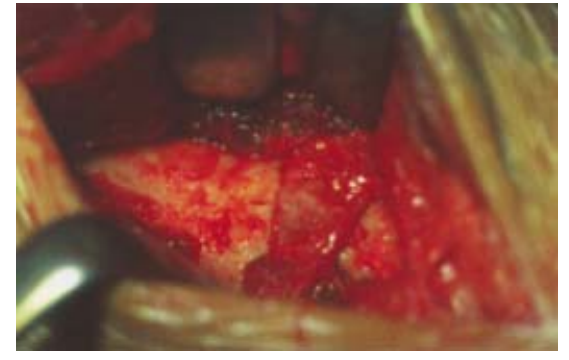

Abb. 5 Distaler Radius mit Darstellung des eingebrachten kortikospongiösen Knochenspans.

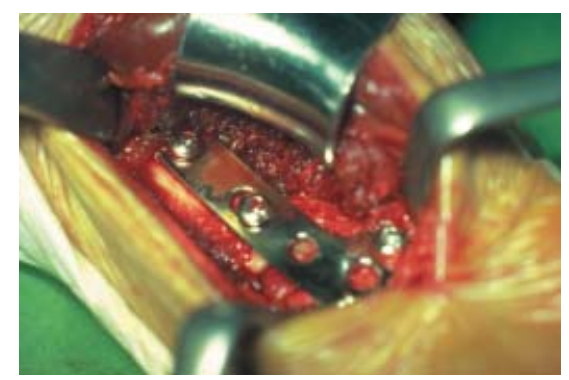

Abb. 6 Distaler Radius mit Darstellung des eingebrachten kortikospongiösen Knochenspans und nach Plattenosteosynthese.

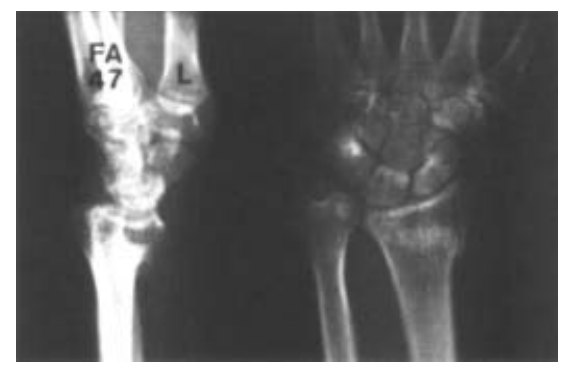

Abb. 7 In Fehlstellung verheilte Flexionsfraktur nach primär konservativer Therapie.

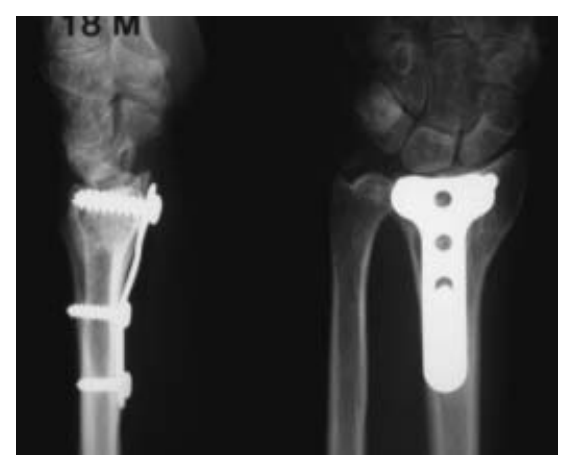

Abb. 818 Monate nach Korrekturosteotomie einer verheilten Flexionsfraktur.
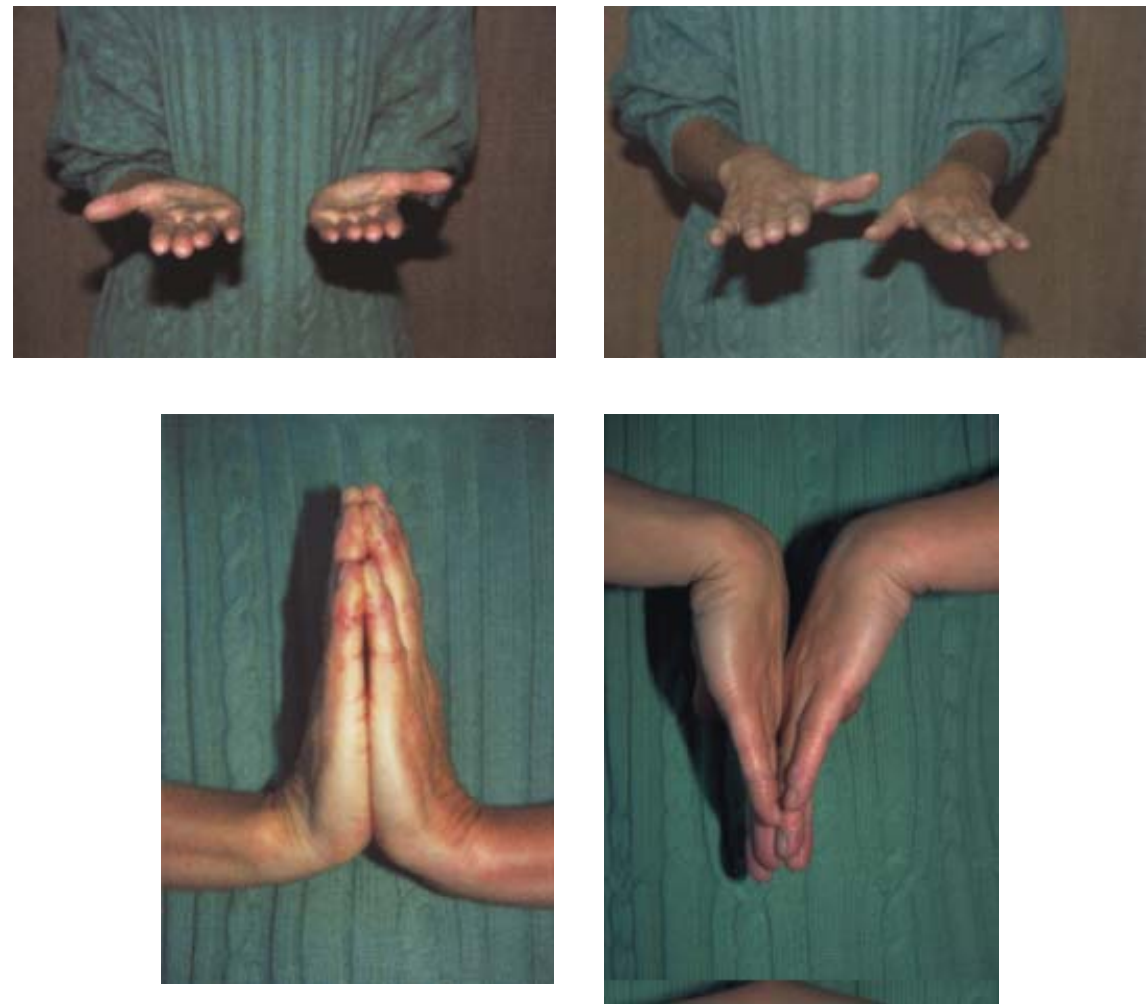

Abb.9-12 Klinische Untersuchung nach Korrekturosteotomie, Fallbeispiel.

durchgeführt. Materialentfernungen von palmaren und ulnaren Implantaten werden vom Alter des Patienten und der Art des Implantates abhängig gemacht.

\section{Ergebnisse}

Vorgestellte Ergebnisse aus der Literatur beziehen sich nur selten auf größere Patientenpopulationen. Trotzdem konnten einige Autoren eindrucksvoll belegen, daß Korrektureingriffe nicht nur eine Besserung der Beweglichkeit, sondern auch der groben Kraft erbrachten und die Schmerzsymptomatik deutlich reduzierten.

Prommersberger [6] berichtete über 91 von 115 nachuntersuchten Patienten mit einer postoperativen Zunahme der Extension-Flexionsbewegung von 26\%, der Ulnar-Radialabduktion von 19\% und Pronation-Supination von $19 \%$.

Die grobe Kraft war durchschnittlich um $38 \%$ gebessert und $69 \%$ der Patienten waren schmerzfrei oder hatten nur leichte Beschwerden.

Abschließend stellt sich noch die Frage nach dem optimalen Zeitpunkt einer Korrekturosteotomie bei Fehlstellungen am distalen Radius. In der Literatur wird ein frühes Vorgehen nach Ausheilung der Fraktur und spätes Vorgehen (bei Beschwerden) unterschieden, wobei die besten Ergebnisse nach 6 bis 9 Monate nach dem Unfall $[4,3]$ erzielt wurden

Zeitpunkt der Korrektur:

- frühes Vorgehen, nach Ausheilung

- spätes Vorgehen, bei Beschwerden

- optimaler Zeitpunkt: 6-9 Monate nach Trauma

\section{Fallbeispiel}

47-jährige Lehrerin, die beim Tanzen auf den linken Arm stürzte und sich eine Flexionsfraktur vom AO-Typ B3 zuzog. Nach primär konservativer Therapie wurde uns die Patientin nach Ausheilung der Fraktur (4. posttraumatischer Monat) in Fehlstellung zur Korrektur vorgestellt. Sie klagte über anhaltende Schmerzen im linken handgelenknahen Unterarm und zeigte eine Extensions-Flexions-Beweglichkeit von 10-0-20 bei aufgehobener Unterarmdrehbeweglichkeit. Röntgenologisch war eine Radiusverkürzung von $11 \mathrm{~mm}$, eine Flexionsfehlstellung der distalen Radiusgelenkfläche von $>25^{\circ}$ sowie eine beginnende Inaktivitätsdystrophie nachweisbar. (Abb. 7 u.8). 
6 Monate nach dem Unfall erfolgte die Korrekturosteotomie am linken distalen Radius über den palmaren Zugang und das Einbringen eines kortikospongiösen Knochenspanes aus dem linken vorderen Beckenkamm. Die Stabilisierung wurde über eine Kleinfragment-T-Platte realisiert, der Unterarmgips nach 3 Wochen entfernt.

Trotz unmittelbar postoperativ begonnenen Pronations-Supinations-Übungen und deutlich gebesserter Beweglichkeit zum Entlassungszeitpunkt, wurde uns die Patientin 8 Wochen nach dem Eingriff erneut vorgestellt. Sie klagte über progrediente ulnarseitige Schmerzen und Bewegungseinschränkungen mit Aufhebung der Rotationsbeweglichkeit in Pronationsstellung. Röntgenologisch zeigten sich eine unveränderte Implantat- und Spanlage sowie achsen- und gelenkgerechte Verhältnisse.

Unter adäquater Analgesie mit Implantation eines Plexuskatheters ließ sich nach physio- sowie ergotherapeutischer Behandlung unter stationären Bedingungen eine nahezu uneingeschränkte Unterarmdrehung (Sup./Pron.: 80-0-80) und Extensions-Flexions-Beweglichkeit von
80-0-70 Grad erreichen. Im Rahmen einer Kontrolluntersuchung waren bei fast schmerzfreier Beweglichkeit und $\mathrm{Zu}-$ nahme der groben Kraft unverändert gute Bewegungsausmaße nachweisbar (Abb.9-12).

\section{Schlussfolgerung}

Das Ziel der Behandlung einer distalen Radiusfraktur besteht in der anatomiegerechten Frakturreposition unter Berücksichtigung von Begleitverletzungen und der richtigen Auswahl befundangepasster Implantate. Kommt es trotzdem zu einer Ausheilung in korrekturbedürftiger Fehlstellung, muss der Eingriff vor Entstehung arthrotischer Veränderungen durchgeführt werden, wobei möglichst physiologische Verhältnisse sowohl radiokarpal als auch im DRUG anzustreben sind. Hierbei sind isolierte Korrektureingriffe an der Ulna nur bei isolierter Radiusverkürzung indiziert. Besteht jedoch eine mehrdimensionale Fehlstellung des distalen Radius und somit auch des DRUG, sollte der Radius in Form und Länge wiederhergestellt werden. Diese Korrektureingriffe unterliegen dabei höchsten Ansprüchen in Vorbereitung, Durchführung und Nachsorge.

\section{Literatur}

1 Adams BD. Effects of radial deformity on distal radioulnar joint mechanics. J Hand Surg 1993; 18A: 492-498

2 Bade H. Lobeck F. Gelenkflächenverhalten der Articulatio radioulnaris distalis bei fehlgestelltem distalen Radius. Unfallchirurgie. 1991: 17: 213-217

3 Jupiter JB, Ring D. A comparson of early and late reconstruction of malunited fractures of the distal end of the radius. J Bone Joint Surg Am 1996; 78 (5): 739- 748

${ }^{4}$ Mollenhoff G, Walz M, Sistermann R. Malposition of healed distal radius fracture. Indication, technique and timing of correction. Handchir Mikorochir Plast Chir 1992; 24 (3): $145-150$

5 Prommersberger K-J, Lanz U. Biomechanik der fehlverheilten distalen Radiusfraktur Eine Literaturübersicht. Handchir Mikrochir Plast Chir 1999; 31: 221 - 226

6 Prommersberger K-J, Moossavi S, Lanz U. Ergebnisse der Korrekturosteotomie fehlverheilter Extensfrakturen der Speiche an typischer Stelle. Handchir Mikrochir Plast Chir 1999; 31: 234-240

7 Schoonhoven J van, Prommersberger K-J, Lanz U. Die Bedeutung des distalen Radioulnargelenks bei rekonstruktiven Eingriffen nach fehlverheilten körperfernen Speichenbrüchen. Orthopäde 1999; 28: 864-871

8 Taleisnik J, Watson HK. Midcarpal instability caused by malunited fractures of the distal radius. J Hand Surg 1984; 9A: 350-357

\section{Dr. med. T. Uzdil}

Assistenzarzt

Prof. Dr. med. K. H. Winker Chefarzt

Klinik für Unfall-, Hand- und Wiederherstellungschirurgie Klinikum Erfurt Nordhäuserstraße 74 99089 Erfurt 